\title{
Modeling Sources of Nonlinearity in a Simple p-i-n Photodetector
}

\author{
Yue Hu, Student Member, IEEE, Brian S. Marks, Member, IEEE, Curtis R. Menyuk, Fellow, IEEE, \\ Vincent J. Urick, Senior Member, IEEE, and Keith J. Williams, Member, IEEE
}

\begin{abstract}
Nonlinearity in p-i-n photodetectors leads to power generation at harmonics of the input frequency, limiting the performance of RF-photonic systems. We use one-dimensional and two-dimensional simulations of the drift-diffusion equations to determine the physical origin of the saturation in a simple heterojunction p-i-n photodetector at room temperature. Incomplete ionization, external loading, impact ionization, and the Franz-Keldysh effect are all included in the model. Impact ionization is the main source of nonlinearity at large reverse bias (>10 V in the device that we simulated). The electron and hole current contributions to the second harmonic power were calculated. We find that impact ionization has a greater effect on the electrons than it does on the holes. We also find that the hole velocity saturates slowly with increasing reverse bias, and the hole current makes a large contribution to the harmonic power at $10 \mathrm{~V}$. This result implies that decreasing the hole injection will decrease the harmonic power.
\end{abstract}

Index Terms-2D simulation, impact ionization, nonlinearity, p-i-n photodetector.

\section{INTRODUCTION}

$\mathbf{N}$ ONLINEARITY in $\mathrm{p}$-i-n photodetectors leads to power generation at harmonics of the input signal frequencies, which limits the performance of RF-photonic systems [1]. The nonlinearity can be measured by integrating an optical signal that carries a single RF frequency and measuring the power in the higher harmonics of that frequency. The harmonic power always decreases as the reverse bias increases up to some voltage, beyond which the harmonic power saturates and can even increase slightly. Determining the origin of this saturation is important for understanding and eliminating the sources of nonlinearity.

Williams et al. [2], [3], Dunn et al. [4], and Walker and Wilson [5] carried out one-dimensional (1D) simulations in the mid-1990s in which they demonstrated that the drift-diffusion equations contain the essential physics that is needed to reproduce this saturation. Williams et al. [2] suggested that p-region

Manuscript received December 20, 2013; revised March 5, 2014; accepted March 28, 2014. Date of publication April 3, 2014; date of current version September 1, 2014.

Y. Hu and C. R. Menyuk are with the Department of Computer Science and Electrical Engineering, University of Maryland Baltimore County, Baltimore, MD, 21250 USA (e-mail: yuehu1@umbc.edu; menyuk@umbc.edu).

B. S. Marks is with the Johns Hopkins Applied Physics Laboratory, Laurel, MD 20723 USA (e-mail: marks@umbc.edu).

V. J. Urick and K. J. Williams are with the Naval Research Laboratory, Washington, DC 20375 USA (e-mail: vincent.urick@nrl.navy.mil; keith williams@nrl.navy.mil).

Color versions of one or more of the figures in this paper are available online at http://ieeexplore.ieee.org.

Digital Object Identifier 10.1109/JLT.2014.2315740 absorption is the dominant source of this saturation. In any $1 \mathrm{D}$ model, it is necessary to assume an effective beam radius for the light beam, which then determines the light intensity. In order to obtain agreement between theory and experiment, Williams et al. [2] used the device radius as the beam radius in the p-region and the much smaller physical beam radius in the intrinsic region.

In this work, we revisit the question of determining the sources of nonlinearity in simple $\mathrm{p}-\mathrm{i}-\mathrm{n}$ photodetectors at room temperature. Subsequent to the early simulation work [2]-[5], it has been discovered that several effects not included in the original studies can play an important role. These include external loading [6], thermionic emission at the heterojunction boundaries [7]-[9], incomplete ionization [9], impact ionization [9]-[12], and the Franz-Keldysh effect [13].

We will show that impact ionization, in particular, is the dominant nonlinear effect at large reverse biases $(>10 \mathrm{~V})$ in the simple device being studied here, leading in most cases to an increase in the harmonic power beyond some voltage. Second, modern-day computers allow us to do cylindrically-symmetric two-dimensional (2D) simulations, and thus take into account transverse diffusion without making ad hoc assumptions about the beam radius. We will show that it is necessary to take into account 2D effects to obtain good agreement with experiments at low reverse biases where the transverse diffusion becomes important. Finally, we carry out fully implicit simulations, in contrast to the explicit simulations in the original work by Williams et al. [2], [3]. Explicit simulations of the drift-diffusion equations are intrinsically unstable [14]. (See also [3], Fig. 4.14, p. 56.]) Williams et al. [2], [3] used a grid-dependent diffusion cap that avoids this instability, but lowers the diffusion by up to a factor of 100 in some regions of the photodetector-in particular, the $\mathrm{p}$-i interface. We did not use a diffusion cap, which in some cases led to different physics for the dominant source of nonlinearity. In particular, we find that the role of p-region absorption is diminished because of diffusive backflow of electrons from the n-region through the intrinsic region to the p-region.

The remainder of this paper is organized as follows: Section II contains a discussion of our photodetector model. Section III describes our computational approach. Section IV contains our simulation results. Section V discusses the sources of nonlinearity in our device. Section VI contains the conclusions.

\section{Photodetector Model}

\section{A. $p$-i-n Photodetector Structure}

The basic structure that we investigate here is a single heterojunction device made from InP and InGaAs [2], as 


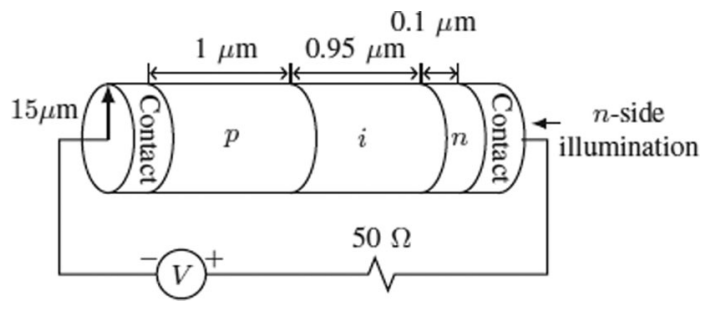

Fig. 1. Structure of the p-i-n photodetector. Lengths are not to scale.

shown in Fig. 1. The device is composed of a highlydoped transparent n-InP substrate of length $w_{n}=0.1 \mu \mathrm{m}$ $\left(N_{D}=2 \times 10^{17} \mathrm{~cm}^{-3}\right)$, an intrinsic layer of n-InGaAs of length $w_{i}=0.95 \mu \mathrm{m} \quad\left(N_{B}=5 \times 10^{15} \mathrm{~cm}^{-3}\right)$, and a degenerately doped p-InGaAs $p$-region of length $w_{p}=1 \mu \mathrm{m}$ $\left(N_{A}=7 \times 10^{18} \mathrm{~cm}^{-3}\right)$, where $N_{A}$ and $N_{D}$ denote the acceptor and donor densities, and $N_{B}$ denotes the unintentional donor density in the intrinsic region. The total length of the photodetector is $L=2.05 \mu \mathrm{m}$. The incident light is assumed to pass through an aperture on the n-side ohmic contact of the device. The device radius is $15 \mu \mathrm{m}$. In the simulation, we set $N_{D}=2 \times 10^{17} \quad \mathrm{~cm}^{-3}, N_{A}=7 \times 10^{18} \quad \mathrm{~cm}^{-3}$, and $N_{B}=5 \times 10^{15} \mathrm{~cm}^{-3}$.

\section{B. Basic Drift-Diffusion Model}

To model the carrier transport in the photodetector, we use both 1D and 2D drift-diffusion models [4], [5], [12], [15]-[21]. This model consists of three equations that govern the dynamics of the electron density $n$, the hole density $p$, and the electric field $\mathbf{E}$ (gradient of the electrostatic potential, $\varphi$ ),

$$
\begin{aligned}
\frac{\partial n}{\partial t} & =G-R(n, p)+\frac{\nabla \cdot \mathbf{J}_{n}}{q}, \\
\frac{\partial p}{\partial t} & =G-R(n, p)-\frac{\nabla \cdot \mathbf{J}_{p}}{q}, \\
\nabla \cdot \mathbf{E} & =\frac{q}{\epsilon}\left(N_{D}^{+}+p-n-N_{A}^{-}\right)
\end{aligned}
$$

where $q$ is the unit of charge (here positive), $G$ and $R$ are the generation and recombination rates, $\epsilon$ is the permittivity of the semiconductor material, and $N_{D}^{+}$and $N_{A}^{-}$are the ionized donor and acceptor impurity concentrations. The variables $\mathbf{J}_{n}$ and $\mathbf{J}_{p}$ are the current densities for electrons and holes, and are given by

$$
\begin{aligned}
& \mathbf{J}_{n}=q n \mathbf{v}_{n}(\mathbf{E})+q D_{n} \nabla n, \\
& \mathbf{J}_{p}=q p \mathbf{v}_{p}(\mathbf{E})-q D_{p} \nabla p
\end{aligned}
$$

where $D_{n}$ and $D_{p}$ are the electron and hole diffusion coefficients, respectively, while $\mathbf{v}_{n}(\mathbf{E})$ and $\mathbf{v}_{p}(\mathbf{E})$ are the electricfield-dependent electron and hole drift velocities, respectively.

The electron velocity as a function of electric field has been measured for InGaAs samples at electric field strengths from $10-100 \mathrm{kV} / \mathrm{cm}$. An empirical expression that has been used to fit $\mathbf{v}_{n}(\mathbf{E})$ for electrons in InGaAs is given by [3], [15]

\begin{tabular}{|c|c|c|}
\hline Parameter & InP & $\mathrm{In}_{0.53} \mathrm{Ga}_{0.47} \mathrm{As}$ \\
\hline $\mathrm{E}_{g}(\mathrm{eV})$ & 1.35 & 0.74 \\
\hline$\chi(\mathrm{eV})$ & 4.38 & 4.6 \\
\hline$\epsilon_{r}(\mathrm{eV})$ & 12.4 & 13.7 \\
\hline$\Delta E_{D}(\mathrm{eV})$ & 0.007 & 0.005 \\
\hline$\Delta E_{A}(\mathrm{eV})$ & 0.025 & 0.025 \\
\hline$\tau_{n, p}$ in $i$-region (ps) & 200 & 200 \\
\hline$\tau_{n, p}$ in doped region (ps) & 2 & 2 \\
\hline$A_{n}\left(\mathrm{~cm}^{-1}\right)$ & $1.12 \times 10^{7}$ & $6.64 \times 10^{7}$ \\
\hline$B_{n}(\mathrm{~V} / \mathrm{cm})$ & $3.11 \times 10^{6}$ & $2 \times 10^{6}$ \\
\hline$A_{p}\left(\mathrm{~cm}^{-1}\right)$ & $4.79 \times 10^{6}$ & $9.34 \times 10^{7}$ \\
\hline$B_{p}(\mathrm{~V} / \mathrm{cm})$ & $2.55 \times 10^{6}$ & $2.26 \times 10^{6}$ \\
\hline$\mu_{n, 0}\left(\mathrm{~cm}^{2} / \mathrm{V} \mathrm{sec}\right)$ & 6000 & 18000 \\
\hline$\mu_{p, 0}\left(\mathrm{~cm}^{2} / \mathrm{V} \mathrm{sec}\right)$ & 180 & 400 \\
\hline$N_{n, \text { ref }}\left(\mathrm{cm}^{-3}\right)$ & $10^{17}$ & $10^{17}$ \\
\hline$N_{p, \text { ref }}\left(\mathrm{cm}^{-3}\right)$ & $6 \times 10^{17}$ & $10^{18}$ \\
\hline$\eta_{n}$ & 0.34 & 0.5 \\
\hline$\eta_{p}$ & 0.64 & 0.45 \\
\hline$\beta\left(\mathrm{cm}^{2} / \mathrm{V}^{2}\right)$ & $8 \times 10^{-8}$ & $8 \times 10^{-8}$ \\
\hline$\gamma$ & 1 & 1 \\
\hline$\alpha\left(\mu \mathrm{m}^{-1}\right)$ & - & 1.15 \\
\hline$v_{n, \mathrm{sat}}(\mathrm{cm} / \mathrm{sec})$ & $10^{7}$ & $10^{7}$ \\
\hline$v_{p, \text { sat }}(\mathrm{cm} / \mathrm{sec})$ & $6 \times 10^{6}$ & $6 \times 10^{6}$ \\
\hline$m_{n}^{*} / m_{0}$ & - & 0.041 \\
\hline$m_{p}^{*} / m_{0}$ & - & 0.59 \\
\hline$D_{0}(\mu \mathrm{m})$ & & 8 \\
\hline$r_{0}(\mu \mathrm{m})$ & & 4 \\
\hline Light Wavelength, $\lambda(\mathrm{nm})$ & & 1319 \\
\hline
\end{tabular}

$$
\mathbf{v}_{n}=\frac{\mathbf{E}\left(\mu_{n}+v_{n, \mathrm{sat}} \beta|\mathbf{E}|\right)}{1+\beta|\mathbf{E}|^{2}}
$$

TABLE I

Material Parameters at $300 \mathrm{~K}$ USED IN THE SimUlation

where $\mu_{n}$ is the electron low-field mobility, $v_{n, \text { sat }}$ is the saturated electron velocity, and $\beta$ is a fitting parameter. The parameters $\mu_{n}, v_{n, \text { sat }}$, and $\beta$ are given in Table I. The hole velocity has also been measured in the range of $50-100 \mathrm{kV} / \mathrm{cm}$. An empirical expression that has been used to fit $\mathbf{v}_{p}(\mathbf{E})$ for the holes in the InGaAs is given by [22]

$$
\mathbf{v}_{p}=\frac{\mu_{p} v_{p, \mathrm{sat}} \mathbf{E}}{\left(v_{p, \mathrm{sat}}^{\gamma}+\mu_{p}^{\gamma}|\mathbf{E}|^{\gamma}\right)^{1 / \gamma}}
$$

where $\mu_{p}$ is the hole low-field mobility, $\gamma$ is an empirical fitting parameter that depends on temperature, and $v_{p \text {,sat }}$ is the saturated hole velocity. The parameters $\mu_{p}, \gamma$, and $v_{p \text {,sat }}$ are given in Table I. In Fig. 2, we show the electron and hole velocities as a function of the electric field magnitude given by (3) and (4).

The doping-dependence of the low-field mobilities, $\mu_{n}$ and $\mu_{p}$, are fitted to available experimental data, by adopting Hilsum's empirical formula [9], [23],

$$
\mu=\frac{\mu_{0}}{1+\left(\frac{N_{D}+N_{A}}{N_{\mathrm{ref}}}\right)^{\eta}}
$$

where $\mu_{0}$ is the mobility in low doping concentration, while $N_{\text {ref }}$ and $\eta$ are empirical parameters. 


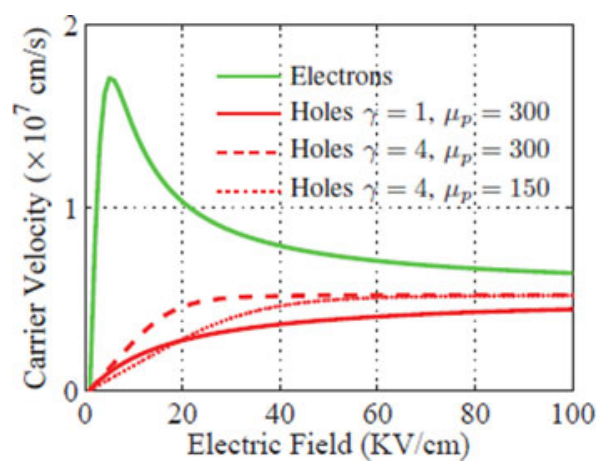

Fig. 2. Empirical expressions for electron and hole velocities versus the electric field from (3) and (4). We use different fitting parameters $\gamma=1,4$ and hole mobility $\mu_{p}=150 \mathrm{~cm}^{2} / \mathrm{V}-\mathrm{s}, 300 \mathrm{~cm}^{2} / \mathrm{V}$-s. This figure is similar [2, Fig. 3].

We use the same expression for the electron and hole diffusion coefficient that Williams [3] used,

$$
\begin{aligned}
D_{n} & =\frac{k T \mu_{n} / q}{\left[1-2\left(|\mathbf{E}| / E_{p}\right)^{2}+\frac{4}{3}\left(|\mathbf{E}| / E_{p}\right)^{3}\right]^{1 / 4}} \\
D_{p} & =\frac{k T}{q} \frac{\mathbf{v}_{p}(\mathbf{E})}{\mathbf{E}},
\end{aligned}
$$

where $E_{p}$ is the electric field at which the diffusion constant peaks. We use $E_{p}=4 \mathrm{kV} / \mathrm{cm}$ in the simulation, with $\mathbf{v}_{p}(\mathbf{E})$ given by (4).

The largest contribution to recombination is the ShockleyRead-Hall (SRH) effect. The expression for SRH recombination is

$$
R=\frac{n p-n_{i}^{2}}{\tau_{p}\left(n+n_{i}\right)+\tau_{n}\left(p+n_{i}\right)}
$$

where $n_{i}$ is the intrinsic density, while $\tau_{p}$ and $\tau_{n}$ are the hole and electron lifetimes, respectively.

In our 2D simulations, we assume that the light intensity incident on the detector is a Gaussian function of the radius and enters through an opening in the n-side, anti-reflection-coated contact. Assuming that there are no reflections, the generation rate as a function of position in the device is expressed as

$$
G(r, z, t)=G_{0}(r, t) \exp \left[-\alpha\left(w_{p}+w_{\mathrm{i}}-z\right)\right]
$$

where $\alpha$ is the absorption coefficient in the InGaAs. As the doping concentration increases, the absorption coefficient decreases, especially when the wavelength of the incident light is close to the energy gap [24]. In the simulation, the absorption coefficient in the p-region is 5\% of that in the i-region [24]. For the harmonic analysis, $G_{0}(r, t)$ is a time harmonic function. We will assume that the beam is Gaussian-shaped with a profile given by

$$
G_{0}(r, t)=G_{0}(t) \exp \left[-2\left(r / r_{0}\right)^{2}\right]
$$

where $G_{0}(t)$ is the time-dependent generation rate and $r_{0}$ is the spot size of the light.

In the 2D model, we use the Gaussian profile in (9). However, in the 1D drift-diffusion model, the physical Gaussian beam profile must be approximated by a constant intensity over an effective beam area. We define an effective beam diameter $D_{0}$ and an average beam intensity $I_{\mathrm{av}}$ such that the total power of this constant approximation is the same as the Gaussian beam, so that

$$
\int_{0}^{\infty} I(r) 2 \pi r d r=I_{\mathrm{av}} \pi\left(\frac{D_{0}}{2}\right)^{2}
$$

where $I(r)=I_{0} \exp \left(-2 r^{2} / r_{0}^{2}\right)$ is the intensity of the Gaussian beam and $r_{0}$ is the $1 / e$ beam radius of the Gaussian beam. In the $1 \mathrm{D}$ simulation, $D_{0}$ is a fitting parameter, and we obtain reasonable results when $D_{0}$ approximately equals $2 r_{0}$. One of the principal advantages of the 2D model is that it is possible to use a realistic beam profile, and it is not necessary to define the fitting parameter $D_{0}$.

The total current output is the sum of the hole and electron currents with the addition of the displacement current integrated over the photodetector length and is given by

$$
I_{\text {total }}=\frac{1}{L} \int_{0}^{L} \int_{0}^{R} 2 \pi r\left(J_{n}+J_{p}+\epsilon \frac{\partial E}{\partial t}\right) d r d z
$$

where $L$ is the length of the device and $R$ is the radius of the device.

To simulate nonlinearities, the photodetector is excited with a constant generation rate until the output current reaches a steady state, at which time a superimposed sinusoidal signal stimulates the device for a number of cycles, typically 10 . We then take the Fourier transform of the output current to obtain the harmonic power. In several cases, we carried the simulations out to 20 cycles and observed no significant difference in the results.

\section{Boundary Conditions and Thermionic Emission}

To determine a set of boundary conditions, the $\mathrm{p}$ - and ncontacts in Fig. 1 are assumed to be ohmic contacts and, as such, offer no barrier to carrier flow. Hence, the carrier densities near the contacts may be approximated by their densities in the bulk region. Assuming thermal equilibrium and vanishing space charge at the ohmic contacts, the boundary conditions at the contacts are

$$
\begin{array}{ll}
p(r, 0)=N_{A}^{-}, & n(r, 0)=\frac{n_{i}^{2}}{p(r, 0)}, \\
n(r, L)=N_{D}^{+}, & p(r, L)=\frac{n_{i}^{2}}{n(r, L)}
\end{array}
$$

where $p(r, 0)$ and $n(r, 0)$ are the hole and electron densities at the contact at $x=0$, while $p(r, L)$ and $n(r, L)$ are the hole and electron densities at the contact at $x=L$.

We set the electrostatic potential at $z=0$ to zero, i.e., $\varphi(r, 0)=0$. We must then set the boundary conditions for $\varphi$ at the other device interfaces. To determine the condition at $\varphi(r, L)$, we must take into account the load resistor. Then, the potential boundary conditions relate the given reverse bias $V_{\mathrm{a}}$, the built-in potential $V_{\mathrm{bi}}$,

$$
V_{\mathrm{bi}}=\frac{k T}{q} \ln \left(\frac{N_{A}^{-} N_{D}^{+}}{n_{i}^{2}}\right)
$$




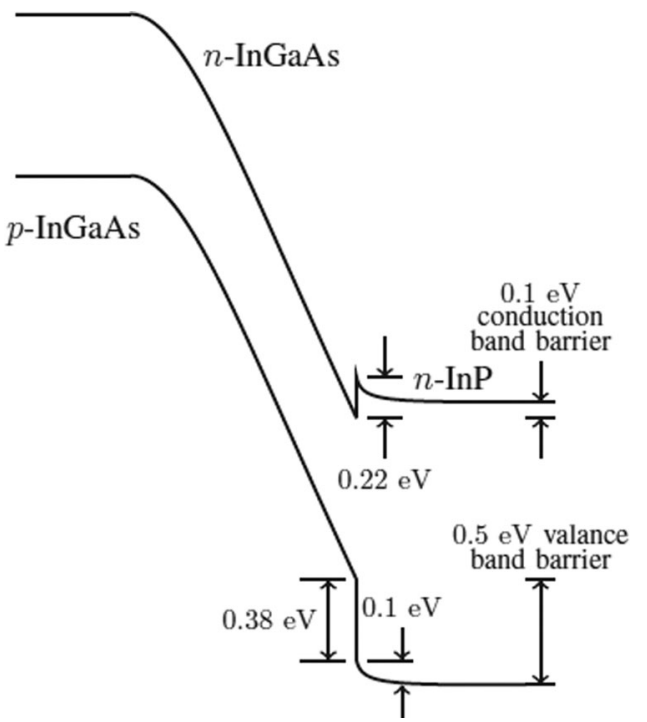

Fig. 3. A depiction of the photodetector band diagram. This figure is similar to [3, Fig. 3.2].

and the current in the photodetector to the electric field in the semiconductor region, so that the boundary condition for the potential is

$$
\varphi(r, L)-\varphi(r, 0)=\varphi(r, L)=V_{\mathrm{a}}-I R_{\mathrm{Load}}+V_{\mathrm{bi}}
$$

where $I$ is the output current. Since the current $I$ is unknown at the start of the simulation, we must find it iteratively. Starting with an initial guess $I_{1}$, we determine a new current $I_{2}$ using (15). We then use $I_{2}$ as the next guess. We iterate until the relative difference is smaller than $10^{-6}$.

At the boundary $r=R$, a floating boundary condition is applied. The boundary conditions are

$$
\begin{array}{ll}
\left.\frac{\partial p}{\partial r}\right|_{r=0}=0, & \left.\frac{\partial p}{\partial r}\right|_{r=R}=0, \\
\left.\frac{\partial n}{\partial r}\right|_{r=0}=0, & \left.\frac{\partial n}{\partial r}\right|_{r=R}=0, \\
\left.\frac{\partial \varphi}{\partial r}\right|_{r=0}=0, & \left.\frac{\partial \varphi}{\partial r}\right|_{r=R}=0 .
\end{array}
$$

Fig. 3 shows the band diagram of the photodetector, where a reverse bias voltage of a few volts is applied to the device. The InGaAs/InP heterojunction depicted in Fig. 3 has a valence band discontinuity of $0.38 \mathrm{eV}$ and a conduction band discontinuity of $0.23 \mathrm{eV}$ [3], [9], [10]. The reduction in the conduction band discontinuity and the increase in the valence band discontinuity of approximately $0.1 \mathrm{eV}$ is the result of the difference in the doping of the intrinsic and $\mathrm{n}$-InGaAs layer. The electrons will be allowed to flow without restriction across the heterojunction because the barrier is only $0.1 \mathrm{eV}$. However, holes are impacted by the $0.5 \mathrm{eV}$ barrier. We use a thermionic emission model [7], [8] to calculate the hole current at the heterojunction. Assuming that $z$ increases from left to right, as shown schematically in
Fig. 1, we write

$$
J_{p}=q v_{1} p_{-} \exp \left[\left(E_{v 1}-E_{v 2}\right) / k T\right]-q v_{2} p_{+}
$$

where $v_{1}$ and $v_{2}$ are respectively the hole emission velocities on the left side and right side of the heterojunction, $p_{-}$and $p_{+}$are the hole densities on the left side and right side of the heterojunction, and $E_{v 1}$ and $E_{v 2}$ are the valance band energies on the left side and right side of the heterojunction.

\section{Additional Effects}

We have already described how we model the external load and thermionic effects in connection with our discussion of the boundary conditions. Here, we discuss how we model incomplete ionization, impact ionization, and the Franz-Keldysh effect. We note that all these effects are modeled at room temperature, which is in accord with the experiments.

1) Incomplete Ionization: The doping impurities introduced into InGaAs and InP are not fully ionized at room temperature [9]. The incomplete ionization of impurities in InGaAs and InP must be considered in a similar manner to those in silicon. Indeed, the impurity energy levels are relatively deep compared to the thermal energy. The model accounts for the incomplete ionization of doping impurities such as boron, aluminium, and nitrogen, using the following expressions:

$$
\begin{aligned}
& N_{D}^{+}=\frac{N_{D}}{1+g_{D} \exp \left(\frac{E_{C}-E_{D}}{k T}\right) \exp \left(\frac{E_{F n}-E_{C}}{k T}\right)}, \\
& N_{A}^{-}=\frac{N_{A}}{1+g_{A} \exp \left(\frac{E_{A}-E_{V}}{k T}\right) \exp \left(-\frac{E_{F p}-E_{V}}{k T}\right)}
\end{aligned}
$$

where $N_{D}^{+}$and $N_{A}^{-}$are the ionized donor and acceptor impurity concentrations, $N_{D}$ and $N_{A}$ are the donor and acceptor impurity concentrations, $g_{D}=2$ and $g_{A}=4$ are the respective ground-state degeneracy of donor and accept impurity levels, $E_{A}$ and $E_{D}$ are the acceptor and donor energy levels, $E_{C}$ and $E_{V}$ are the low conduction band and the high valence band energy levels, $E_{F n}$ and $E_{F p}$ are the quasi-Fermi energy levels for the electrons and holes, $k$ is the Boltzmann constant, and $T$ is the temperature. The energy differences in the simulation are $\Delta E_{D}=E_{C}-E_{D}=5 \mathrm{meV}$ and $\Delta E_{A}=E_{A}-E_{V}=25$ $\mathrm{meV}$. The basic variables in the drift-diffusion equations, (1), are the potential (or electric field), the electron concentration, and the hole concentration. Therefore, it is more convenient to rewrite (18) in terms of the carrier concentration instead of the quasi-Fermi levels:

$$
N_{D}^{+}=\frac{N_{D}}{1+g_{D} n / n_{1}}, \quad N_{A}^{-}=\frac{N_{A}}{1+g_{A} p / p_{1}}
$$

where

$$
\begin{aligned}
& n_{1}=N_{C} \exp \left(-\frac{\Delta E_{D}}{k_{B} T}\right), \\
& p_{1}=N_{V} \exp \left(-\frac{\Delta E_{A}}{k_{B} T}\right)
\end{aligned}
$$


and we make use of the expressions

$$
\begin{aligned}
& n=N_{C} \exp \left(\frac{E_{F n}-E_{C}}{k_{B} T}\right), \\
& p=N_{V} \exp \left(-\frac{E_{F p}-E_{V}}{k_{B} T}\right) .
\end{aligned}
$$

2) Impact Ionization: In a strong electric field, accelerating electrons and holes can have kinetic energies that are larger than the band gap (around $1 \mathrm{eV}$ for $\mathrm{In}_{0.53} \mathrm{Ga}_{0.47} \mathrm{As}[25]$ ), at which point a lattice collision will produce an electron-hole pair. The electron and hole move in opposite directions and can themselves produce new electron-hole pairs. With a strong electric field and an acceleration region with a sufficiently long length, this mechanism can lead to avalanche breakdown [26]. In the $\mathrm{p}-\mathrm{i}-\mathrm{n}$ photodetectors that we are considering, the acceleration lengths are too small to lead to avalanche breakdown since electrons leave the acceleration region too quickly. Nonetheless, this impact ionization can lead to an important increase in the electron and hole densities. We may write the electron and hole generation rate $G_{i}$ as

$$
G_{i}=\alpha_{n} \frac{\left|\mathbf{J}_{n}\right|}{q}+\alpha_{p} \frac{\left|\mathbf{J}_{p}\right|}{q}
$$

where $\alpha_{n}$ and $\alpha_{p}$ are the impact ionization coefficients of the electrons and holes, respectively. We calculate their values using the formula [9]:

$$
\begin{aligned}
& \alpha_{n}=A_{n} \cdot \exp \left[-\left(\frac{B_{n}}{|\mathbf{E}|}\right)^{m}\right], \\
& \alpha_{p}=A_{p} \cdot \exp \left[-\left(\frac{B_{p}}{|\mathbf{E}|}\right)^{m}\right]
\end{aligned}
$$

where $A_{n}, B_{n}, A_{p}$, and $B_{p}$ are experimentally determined parameters [9], [10]. The exponent $m$ is taken to be 1.05. The values that we use, shown in Table I for InGaAs, are modified slightly from the values in [9], [10] in order to obtain harmonic powers that are consistent with experiments. However, all values fall within the range of experimental error.

3) Franz-Keldysh Effect: When the photon energy of the incident optical light is close to the band edge of the InGaAs absorber, the Franz-Keldysh effect must be taken into account [27]. The Franz-Keldysh effect leads to oscillations in the carrier transition probability for energies that are greater than the band gap and tunneling of the electron state into the forbidden band due to band-bending below the band gap in the presence of an applied electric field. The absorption coefficient is given by [28]

$$
\alpha(\omega, E)=\left(C \theta_{F}^{1 / 2} / \omega\right)\left[\left|\frac{d \operatorname{Ai}(\beta)}{d \beta}\right|^{2}-\beta|\operatorname{Ai}(\beta)|^{2}\right]
$$

where $\omega$ is the light frequency, $C$ is a fitting parameter, and $\operatorname{Ai}(x)$ is the Airy function. We also have

$$
\beta=\frac{\omega_{1}-\omega}{\theta_{F}}
$$

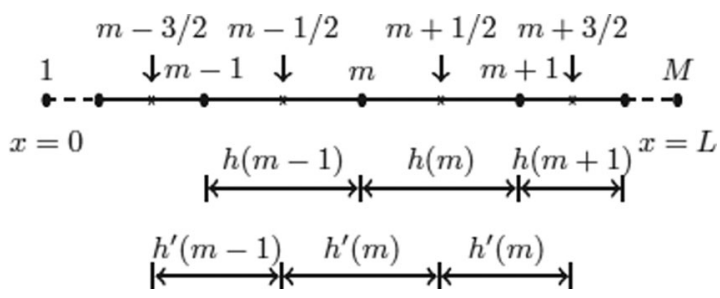

Fig. 4. Numerical mesh used for the finite difference spatial discretization of the 1-D drift-diffusion equation.

and

$$
\left(\theta_{F}\right)^{3}=\frac{e^{2} E^{2}}{2 m_{n p} \hbar}
$$

where $\hbar \omega_{1}$ is the energy of the band gap, and $m_{n p}$ is the reduced mass of the electric-hole pair. We may write

$$
\frac{1}{m_{n p}}=\frac{1}{m_{n}^{*}}+\frac{1}{m_{p}^{*}}
$$

where $m_{n}^{*}$ and $m_{p}^{*}$ are the electron and hole effective masses, respectively.

\section{COMPutational Model}

\section{A. 1D Computational Model}

When discretizing the drift-diffusion equations for numerical computation, it is important to use a fully implicit method [14]. Explicit methods are intrinsically unstable and thus require an unreasonably small time step to yield physical results with the diffusion coefficients given in (6). In our simulations, the time step would be smaller than $10^{-15} \mathrm{~s}$ if we use an explicit method, compared with a time step of $10^{-12} \mathrm{~s}$ using an implicit method. We have used the implicit Euler method to discretize the equations in time $t$. We use second-order finite differences to discretize the spatial dimension $x$. We discretize the $x$-dimension using the mesh shown schematically in Fig. 4. We define $p, n$, and $\varphi$, at integral points in the mesh that are indexed by $m=1,2, \ldots, M$. The current and electric field are defined at intermediate points that are indexed by $m=3 / 2,5 / 2, \ldots, M-1 / 2$. We use an uneven spacing between mesh points, and hence we specify the distance between the integral points $m$ and $m+1$ as $h(m)$, and the distance between the intermediate points $m-1 / 2$ and $m+1 / 2$ as $h^{\prime}(m)$. We set

$$
h^{\prime}(m)=\frac{h(m-1)+h(m)}{2} .
$$

Using this mesh, (1) and (2) become

$$
\begin{aligned}
\frac{n_{m}^{i+1}-n_{m}^{i}}{\delta t}= & \frac{1}{q} \frac{\left(J_{n}\right)_{m+1 / 2}^{i+1}-\left(J_{n}\right)_{m-1 / 2}^{i+1}}{h^{\prime}(m)} \\
& +G_{m}^{i+1}+G_{i m}^{i+1}-R_{m}^{i+1}, \\
\frac{p_{m}^{i+1}-p_{m}^{i}}{\delta t}= & -\frac{1}{q} \frac{\left(J_{p}\right)_{m+1 / 2}^{i+1}-\left(J_{p}\right)_{m-1 / 2}^{i+1}}{h^{\prime}(m)} \\
& +G_{m}^{i+1}+G_{i m}^{i+1}-R_{m}^{i+1},
\end{aligned}
$$




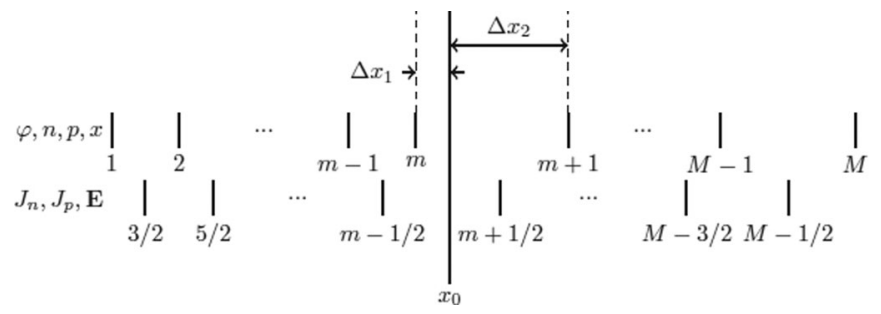

Fig. 5. Gridding scheme used in the device model for multilayer devices.

$$
\begin{array}{r}
\frac{1}{h^{\prime}(m)}\left[\frac{\varphi_{m+1}^{i+1}-\varphi_{m}^{i+1}}{h(m)}-\frac{\varphi_{m}^{i+1}-\varphi_{m-1}^{i+1}}{h(m-1)}\right] \\
=-\frac{q}{\epsilon}\left(N_{D m}^{+}-N_{A m}^{-}+p_{m}^{i+1}-n_{m}^{i+1}\right)
\end{array}
$$

and

$$
\begin{aligned}
\left(J_{n}\right)_{m+1 / 2}^{i+1}= & q\left(D_{n}\right)_{m+1 / 2}^{i+1} \frac{n_{m+1}^{i+1}-n_{m}^{i+1}}{h(m)} \\
& +q\left(v_{n}\right)_{m+1 / 2}^{i+1} \frac{n_{m+1}^{i+1}+n_{m}^{i+1}}{2}, \\
\left(J_{p}\right)_{m+1 / 2}^{i+1}= & -q\left(D_{p}\right)_{m+1 / 2}^{i+1} \frac{p_{m+1}^{i+1}-p_{m}^{i+1}}{h(m)} \\
& +q\left(v_{p}\right)_{m+1 / 2}^{i+1} \frac{p_{m+1}^{i+1}+p_{m}^{i+1}}{2}
\end{aligned}
$$

where $n_{m}^{i+1}$ and $p_{m}^{i+1}$ are the electron and hole densities at the point $m$ and time-step $i+1$, respectively, while $\left(J_{n}\right)_{m+1 / 2}^{i+1}$ and $\left(J_{p}\right)_{m+1 / 2}^{i+1}$ are the electron and hole currents at the point $m+$ $1 / 2$ and time-step $i+1$, respectively, $G_{m}^{i+1}$ is the generation rate at the point $m$ and time-step $i+1, R_{m}^{i+1}$ is the recombination rate at the point $m$ and time-step $i+1, \varphi_{m}^{i+1}$ is the electrostatic potential at the point $m$ and time-step $i+1, N_{D m}^{+}$ and $N_{A m}^{-}$are the ionized donor and acceptor doping density at the point $m,\left(D_{n}\right)_{m+1 / 2}^{i+1}$ and $\left(D_{p}\right)_{m+1 / 2}^{i+1}$ are the electron and hole diffusion coefficient at the point $m+1 / 2$ and time-step $i+1,\left(v_{n}\right)_{m+1 / 2}^{i+1}$ and $\left(v_{p}\right)_{m+1 / 2}^{i+1}$ are the electron and hole velocities at the point $m+1 / 2$ and time-step $i+1$. Here, we used the average carrier densities $\left(n_{m+1}^{i+1}+n_{m}^{i+1}\right) / 2$ and $\left(p_{m+1}^{i+1}+p_{m}^{i+1}\right) / 2$ to approximate the carrier densities $n_{m+1 / 2}^{i+1}$ and $p_{m+1 / 2}^{i+1}$ at the intermediate $m+1 / 2$ point and time-step $i+1$ in (30).

At the heterojunction interface $x_{0}$ shown in Fig. 5, the discretization is different. We treat the drift-diffusion equation as a differential equation in the hole density, assuming all other variables are constant across the grid, and integrating across the grid element to obtain the hole density at the interface $x_{0}$. For a grid element containing a heterojunction interface, there is a discontinuity in the hole density at this interface. So, the integration is done in two steps, first from $x(m)$ to $x_{0}$ and then from $x_{0}$ to $x(m+1)$, as illustrated in Fig. 5. The drift-diffusion

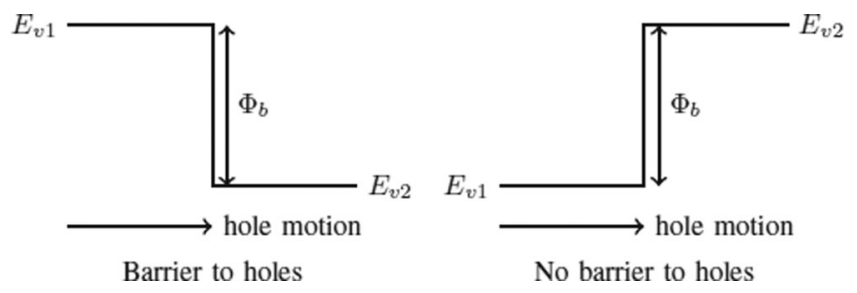

Fig. 6. Schematic of the boundary condition used at the heterojunction.

equation for holes, which is obtained by inserting (6) into (2b), can be written as a differential equation in the hole density,

$$
\frac{\partial p}{\partial x}=\frac{q E}{k T} p-\frac{E J_{p}}{k T v_{p}} .
$$

Integrating from $x(m)$ to $x_{0}$ yields

$$
\begin{aligned}
p\left(x_{0}^{-}\right)= & p(m) \exp \left[\frac{q E(m+1 / 2)}{k T} \Delta x_{1}\right]-\frac{J_{p}(m+1 / 2)}{q v_{p}(m+1 / 2)} \\
& \times\left\{1-\exp \left[\frac{q E(m+1 / 2)}{k T} \Delta x_{1}\right]\right\} .
\end{aligned}
$$

The integral from $x_{0}$ to $x(m+1)$ yields:

$$
\begin{aligned}
p\left(x_{0}^{+}\right)= & p(m+1) \exp \left[-\frac{e E(m+1 / 2)}{k T} \Delta x_{2}\right] \\
& -\frac{J_{p}(m+1 / 2)}{q v_{p}(m+1 / 2)}\left\{1-\exp \left[-\frac{q E(m+1 / 2)}{k T} \Delta x_{2}\right]\right\} .
\end{aligned}
$$

Thermionic emission [7], [8] is considered at the heterojunction interface. The possible barriers for holes are illustrated in Fig. 6. When holes move from a material with a higher valance band energy to a lower valance band energy, as shown on the left of Fig. 6, the heterojunction is a barrier to holes, which is the case in our structure. When holes move from a material with a lower valance band energy to a material with a higher valance band energy, as shown on the right of Fig. 6, then holes move freely through the heterojunction. This kind of heterojunction is not a barrier to holes. This case does not appear in our structure. Using (17), the current at the heterojunction can be written as

$$
\begin{aligned}
J_{p}(m+1 / 2)= & q v_{1} p\left(x_{0}^{-}\right) \exp \left[\left(E_{v 1}-E_{v 2}\right) / k T\right] \\
& -q v_{2} p\left(x_{0}^{+}\right) .
\end{aligned}
$$

Inserting (32) and (33) into (34) and rearranging yields

$$
J_{p}(m+1 / 2)=q v_{p}(m+1 / 2) \frac{A-B}{1+C-D}
$$

where $A, B, C$, and $D$ are

$$
\begin{aligned}
& A=p(m) \exp \left[\frac{q E(m+1 / 2) \Delta x 1+E_{v 1}-E_{v 2}}{k T}\right], \\
& B=p(m+1) \exp \left[-\frac{q E(m+1 / 2) \Delta x_{2}}{k T}\right],
\end{aligned}
$$




$$
\begin{aligned}
& C=\left\{1-\exp \left[\frac{q E(m+1 / 2) \Delta x_{1}}{k T}\right]\right\} \exp \left[\frac{E_{v 1}-E_{v 2}}{k T}\right], \\
& D=1-\exp \left[-\frac{q E(m+1 / 2) \Delta x_{2}}{k T}\right] .
\end{aligned}
$$

We have assumed that the electric field and the hole velocity are the same across the heterojunction interface.

\section{B. 2D Computational Model}

We have used the implicit Euler method to discretize the equations in time $t$. We use a second-order finite difference scheme to discretize the spatial dimensions $r$ and $z$. The mesh is shown schematically in Fig. 7. The integral points in the mesh, labeled $(i, j)$, are where the values of $p, n$, and $\varphi$ are defined, while the intermediate points labeled $(i-1 / 2, j)$ and $(i, j-1 / 2)$ are where the current and electric field are defined. We use an uneven spacing between mesh points, and hence we specify the distance between the integral points $(i, j)$ and $(i, j-$ $1)$ as $h_{j-1}$ in the longitudinal ( $\left.z\right)$ direction, and between $(i, j)$ and $(i-1, j)$ as $k_{i-1}$ in the radial $(r)$ direction. The distance between the intermediate point $(i, j+1 / 2)$ and $(i, j-1 / 2)$ is $h_{j}^{\prime}$ and between the intermediate point $(i+1 / 2, j)$ and $(i-$ $1 / 2, j)$ is $k_{i}^{\prime}$. We set

$$
h_{j}^{\prime}=\frac{h_{j-1}+h_{j}}{2}, \quad k_{i}^{\prime}=\frac{k_{j-1}+k_{j}}{2} .
$$

Our time step is given by $\delta t$. We thus obtain

$$
\begin{aligned}
\frac{n_{i, j}^{t+1}-n_{i, j}^{t}}{\delta t}= & \frac{1}{q} \frac{\left(J_{n r}\right)_{i+1 / 2, j}^{t+1}-\left(J_{n r}\right)_{i-1 / 2, j}^{t+1}}{k_{i}^{\prime}}+\frac{1}{q} \frac{\left(J_{n r}\right)_{i, j}^{t+1}}{r_{i}} \\
& +\frac{1}{q} \frac{\left(J_{n z}\right)_{i, j+1 / 2}^{t+1}-\left(J_{n z}\right)_{i, j-1 / 2}^{t+1}}{h_{j}^{\prime}} \\
& +G_{i, j}^{t+1}+G_{i i, j}^{t+1}-R_{i, j}^{t+1}, \\
\frac{p_{i, j}^{t+1}-p_{i, j}^{t}}{\delta t}= & -\frac{1}{q} \frac{\left(J_{p r}\right)_{i+1 / 2, j}^{t+1}-\left(J_{p r}\right)_{i-1 / 2, j}^{t+1}}{k_{i}^{\prime}}-\frac{1}{q} \frac{\left(J_{p r}\right)_{i, j}^{t+1}}{r_{i}} \\
& -\frac{1}{q} \frac{\left(J_{p z}\right)_{i, j+1 / 2}^{t+1}-\left(J_{p z}\right)_{i, j-1 / 2}^{t+1}}{h_{j}^{\prime}} \\
& +G_{i, j}^{t+1}+G_{i i, j}^{t+1}-R_{i, j}^{t+1}, \\
& -\frac{q}{\epsilon}\left(N_{D i, j}^{+}-N_{A i, j}^{-}+p_{i, j}^{t+1}-n_{i, j}^{t+1}\right) \\
= & \frac{1}{k_{i}^{\prime}}\left[\frac{\varphi_{i+1, j}-\varphi_{i, j}}{k_{i}}-\frac{\varphi_{i, j}-\varphi_{i-1, j}}{k_{i-1}}\right] \\
& +\frac{1}{h_{j}^{\prime}}\left[\frac{\varphi_{i, j}+1}{h_{j}}-\frac{\varphi_{i, j}}{h_{j-1}}\right] \varphi_{i, j-1} \\
& +\frac{1}{r_{i}} \frac{\varphi_{i+1, j}-\varphi_{i-1, j}}{k_{i}+k_{i-1}}
\end{aligned}
$$

and

$$
\begin{aligned}
\left(J_{n r}\right)_{i+1 / 2, j}^{t+1}= & q\left(D_{n}\right)_{i+1 / 2, j}^{t+1} \frac{n_{i+1, j}^{t+1}-n_{i, j}^{t+1}}{k_{i}} \\
& +q\left(v_{n r}\right)_{i+1 / 2, j}^{t+1} \frac{n_{i+1, j}^{t+1}+n_{i, j}^{t+1}}{2} \\
\left(J_{n r}\right)_{i, j}^{t+1}= & q\left(D_{n}\right)_{i, j}^{t+1} \frac{n_{i+1, j}^{t+1}-n_{i-1, j}^{t+1}}{k_{i}+k_{i-1}} \\
& +q\left(v_{n r}\right)_{i, j}^{t+1} n_{i, j}^{t+1}, \\
\left(J_{n z}\right)_{i, j+1 / 2}^{t+1}= & q\left(D_{n}\right)_{i, j+1 / 2}^{t+1} \frac{n_{i, j+1}^{t+1}-n_{i, j}^{t+1}}{h_{j}} \\
& +q\left(v_{n z}\right)_{i, j+1 / 2}^{t+1} \frac{n_{i, j+1}^{t+1}+n_{i, j}^{t+1}}{2} \\
\left(J_{p r}\right)_{i+1 / 2, j}^{t+1}= & -q\left(D_{p}\right)_{i+1 / 2, j}^{t+1} \frac{p_{i+1, j}^{t+1}-p_{i, j}^{t+1}}{k_{i}} \\
& +q\left(v_{p}\right)_{i+1 / 2, j}^{t+1} \frac{p_{i+1, j}^{t+1}+p_{i, j}^{t+1}}{2} \\
& +q\left(v_{p}\right)_{i, j+1 / 2}^{t+1} \frac{p_{i, j+1}^{t+1}+p_{i, j}^{t+1}}{2} \\
\left(J_{p r}\right)_{i, j}^{t+1}= & -q\left(D_{p}\right)_{i, j}^{t+1} \frac{p_{i+1, j}^{t+1}-p_{i-1, j}^{t+1}}{k_{i}+k_{i-1}} \\
& +q\left(v_{p}\right)_{i, j}^{t+1} p_{i, j}^{t+1}, \\
\left(J_{p z}\right)_{i, j+1 / 2}^{t+1}= & -q\left(D_{p}\right)_{i, j+1 / 2}^{t+1} \frac{p_{i, j+1}^{t+1}-p_{i, j}^{t+1}}{h_{j}} \\
&
\end{aligned}
$$

where $n_{i, j}^{t+1}$ and $p_{i, j}^{t+1}$ are the electron and hole densities at the point $(i, j)$ and time-step $t+1$, respectively, while $\left(J_{n r}\right)_{i+1 / 2, j}^{t+1}$ and $\left(J_{p r}\right)_{i+1 / 2, j}^{t+1}$ are the electron and hole currents at the point $(i+1 / 2, j)$ and time-step $t+1$ in the radial direction, respectively, $\left(J_{n z}\right)_{i, j+1 / 2}^{t+1}$ and $\left(J_{p z}\right)_{i, j+1 / 2}^{t+1}$ are the electron and hole currents at the point $(i, j+1 / 2)$ and time-step $t+1$ in the longitudinal direction, respectively, $G_{i, j}^{t+1}$ is the generation rate at the point $(i, j)$ and time-step $t+1, R_{i, j}^{t+1}$ is the recombination rate at the point $(i, j)$ and time-step $t+1, G_{i, j}^{t+1}$ is the impact ionization generation rate at the point $(i, j)$ and timestep $t+1, \varphi_{i, j}$ is the electrostatic potential at the point $(i, j)$ and time-step $t+1, N_{D i, j}^{+}$and $N_{A i, j}^{-}$are the ionized donor and acceptor doping density at the point $(i, j),\left(D_{n}\right)_{i+1 / 2, j}^{t+1}$ and $\left(D_{p}\right)_{i+1 / 2, j}^{t+1}$ are the electron and hole diffusion coefficient at the point $(i+1 / 2, j)$ and time-step $t+1,\left(D_{n}\right)_{i, j+1 / 2}^{t+1}$ and $\left(D_{p}\right)_{i, j+1 / 2}^{t+1}$ are the electron and hole diffusion coefficient at the point $(i, j+1 / 2)$ and time-step $t+1,\left(v_{n r}\right)_{i+1 / 2, j}^{t+1}$ and $\left(v_{p r}\right)_{i+1 / 2, j}^{t+1}$ are the electron and hole velocities at the point $(i+1 / 2, j)$ and time-step $t+1$ in the radial direction, $\left(v_{n z}\right)_{i, j+1 / 2}^{t+1}$ and $\left(v_{p z}\right)_{i, j+1 / 2}^{t+1}$ are the electron and hole velocities at the point $(i+1 / 2, j)$ and time-step $t+1$ in the longitudinal direction. 


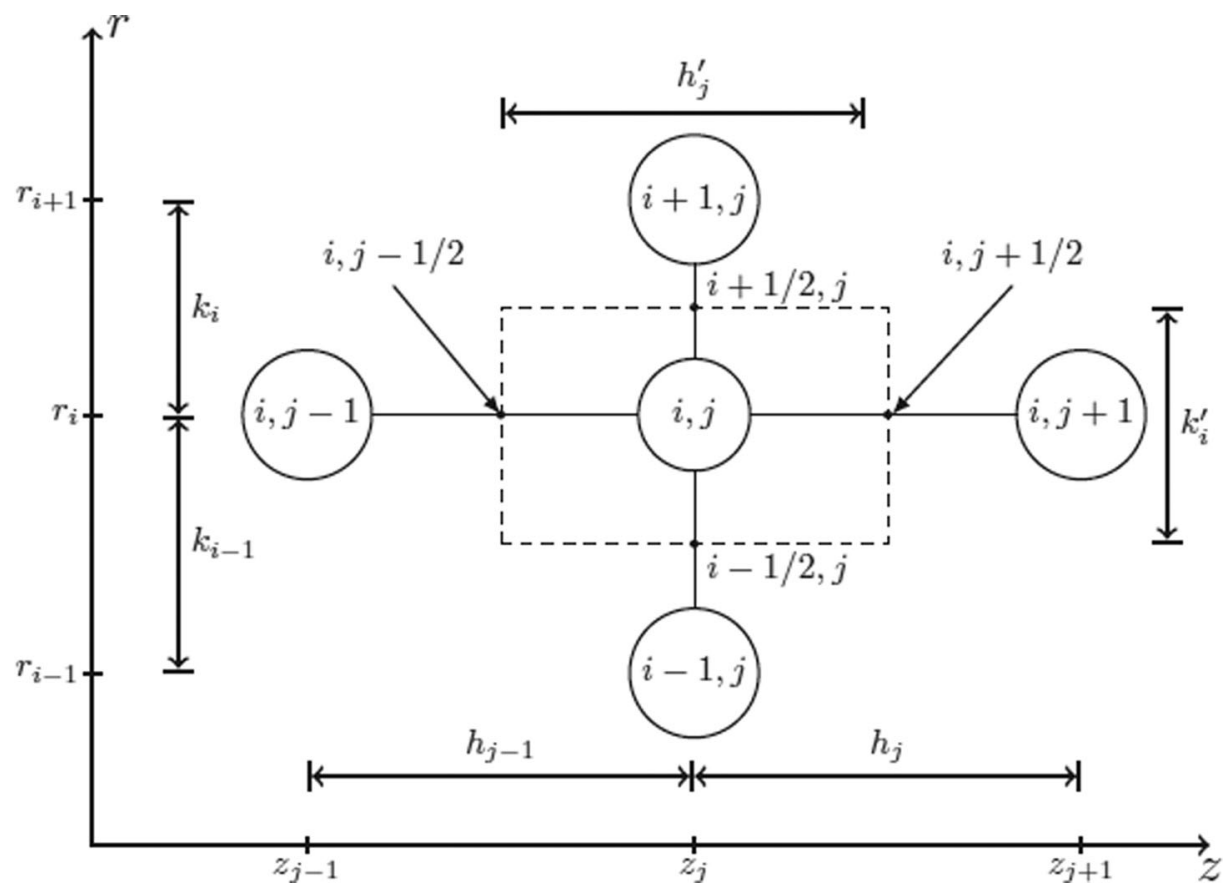

Fig. 7. Numerical mesh used for the finite difference spatial discretization of the $2 \mathrm{D}$ drift-diffusion equation.

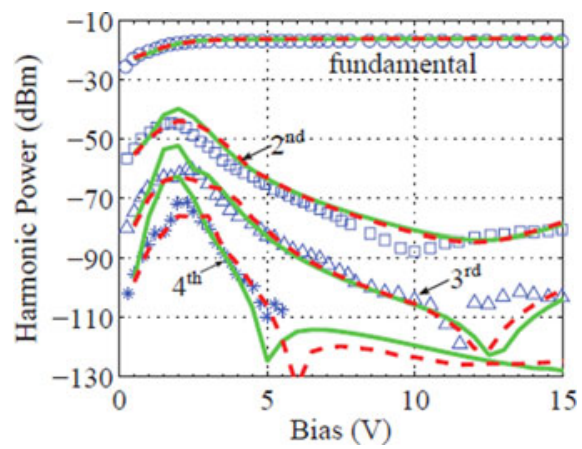

Fig. 8. Measured (symbols) and calculated harmonic power. The green solid and red dashed curves show the results of the $1 \mathrm{D}$ and 2D models.

\section{Computational Results}

The parameters used in our simulations are shown in Table I. In order to see how these parameter affect the results, some parameters are adjusted. In the following simulations, we use the parameters shown in Table I unless otherwise specified.

\section{A. Comparison Between the $1 D$ and 2D Models}

The 1D model and 2D models are compared in Fig. 8. In order to match the experimental data, we set $D_{0}=8 \mu \mathrm{m}$ in the 1D simulations and we set the spot size of the Gaussian beam width equal to $4 \mu \mathrm{m}$ in the $2 \mathrm{D}$ simulations. Compared to the $1 \mathrm{D}$ results, the harmonic power in the $2 \mathrm{D}$ results is lower when the reverse bias is small. This difference comes from the assumption of a constant generation rate as a function of $r$, which is implicit in the 1D model. When $r$ is small, the generation rate in the 2D model must be higher than the constant generation rate in the 1D model, which will induce large harmonic powers. However, when $r$ is large, the generation rate in the $2 \mathrm{D}$ model becomes smaller than in the 1D model inducing carrier flow in the radial direction. This transverse flow is particularly important at low reverse bias, and we find the largest discrepancies between the $1 \mathrm{D}$ and 2D models where the reverse bias is less than $3 \mathrm{~V}$.

Despite the discrepancies between the 1D and 2D models as well as the necessity of empirically determining the parameter $D_{0}$ in the 1D model so that the results best match the experiments, it has the important advantage that 1D model is far more rapid computationally than $2 \mathrm{D}$ simulations. To calculate the data in the 1D simulations in Fig. 8 took $30 \mathrm{~min}$ of run time on a Dell T3500 computer, while the data in the $2 \mathrm{D}$ simulation would have taken $60 \mathrm{~h}$ of run time on the same computer that we used for the 1D simulations. Instead we used UMBC's High Performance Computing Facility (HPCF) [http://www.umbc.edu/hpcf/], which required $6 \mathrm{~h}$ of run time.

\section{B. External Loading and Thermionic Emission}

External loading is another source of nonlinearity in the photodetector. The simulation results are shown in Fig. 9. The results show that external loading only makes a small difference in the harmonic powers.

We expect that external loading has its greatest effect on the harmonic power when the reverse bias is low. However, it affects the harmonic power elsewhere. First, the load resistor reduces the bias applied to the device. For an average current of $1 \mathrm{~mA}$, the potential across the resistor is $0.05 \mathrm{~V}$ for a $50 \Omega$ resistor. So, the harmonic power as a function of reverse bias should shift to lower bias. Second, an external load is also a source of nonlinearity. When the generation increases in the device, there is more space charge in the depletion region, so that the electric field in the depletion region changes, leading to space-charge induced nonlinearity [2]. When there is a large 


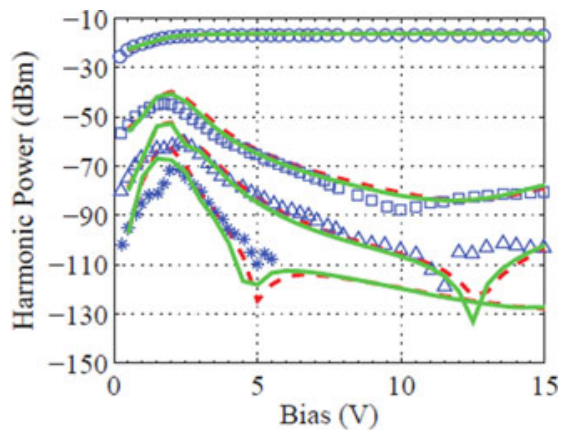

(a)

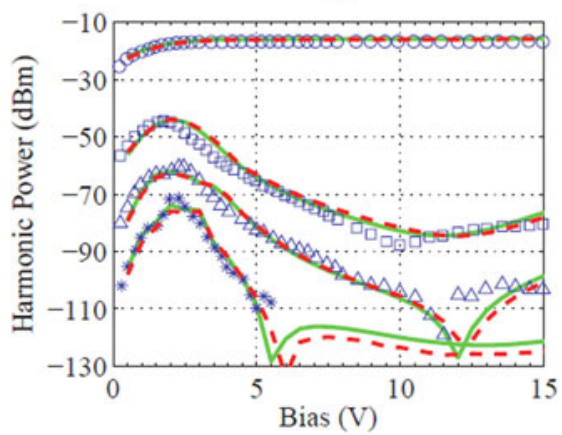

(b)

Fig. 9. Measured and calculated harmonic powers. We compare the load resistor's influence to the harmonic power of the photodetector. The red dashed curves show the results without the load resistor, and the green solid curves show the results of keeping a $50 \Omega$ resistor in the simulation. (a) 1D model. (b) 2D model.

generation, the current and the bias across the resistor will also increase. So, there is less reverse bias on the photodetector, and the electric field decreases, changing the current.

In our simulations, the load resistor is not significant because the output current of $1 \mathrm{~mA}$ is too small to lead to a significant voltage drop across the resistor. The drop is only $0.05 \mathrm{~V}$. However, we note that the load resistor becomes important when the current is large as is the case in some modern-day devices [11], [13].

When thermionic boundary conditions are replaced by a complete barrier, as was done by Williams, et al. [3], no difference is observed in the results. So the comparison between these two models is not shown.

\section{Impact Ionization}

In Fig. 10, we show the effect of impact ionization. Below $10 \mathrm{~V}$, it makes little to no difference in the results. However, above $10 \mathrm{~V}$, the results are significant, leading in most cases to an increase in the harmonic power as the reverse bias increases. It is not possible to get agreement with experiments without including this effect.

\section{Franz-Keldysh Effect}

In all the simulations that we have shown thus far, we have not included the Franz-Keldysh effect. We show the results when this effect is included in Fig. 11. In both the 1D and 2D models, it leads to a rapid and unphysical oscillation in the har-

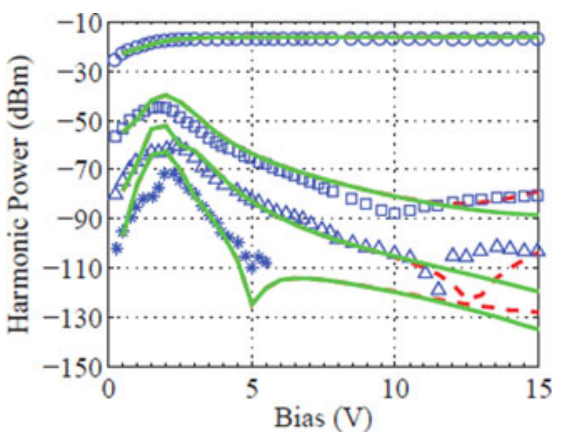

(a)

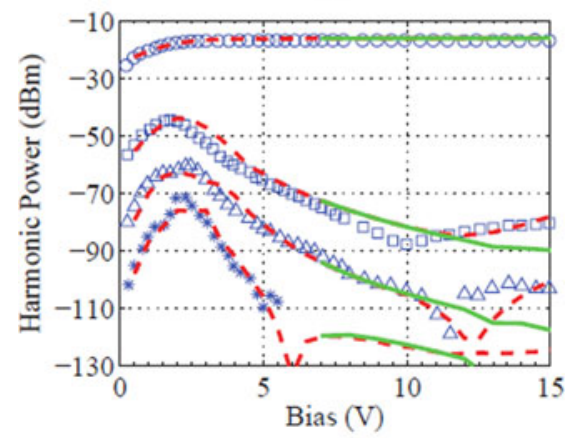

(b)

Fig. 10. Measured and calculated harmonic powers of the photodetector output current as a function of the reverse bias. Impact ionization and a load resistor are kept in the simulations. The harmonic power is compared with (red dashed curve) and without (green solid curve) impact ionization. The red dashed curve is not visible until almost $10 \mathrm{~V}$ in Fig. 10(a) because the green solid curve lies on top of it. In Fig. 10(b), we only plot the green solid curve above 7 V. (a) 1D model. (b) $2 \mathrm{D}$ model.

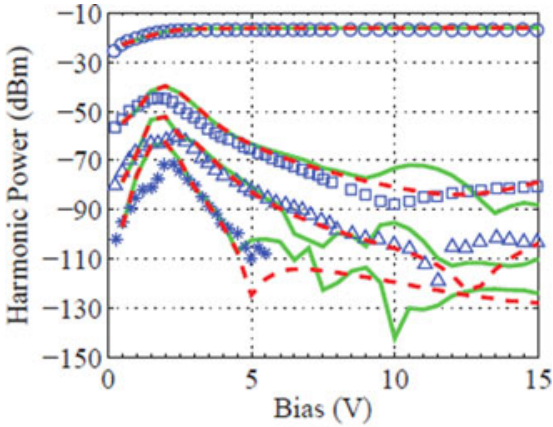

(a)

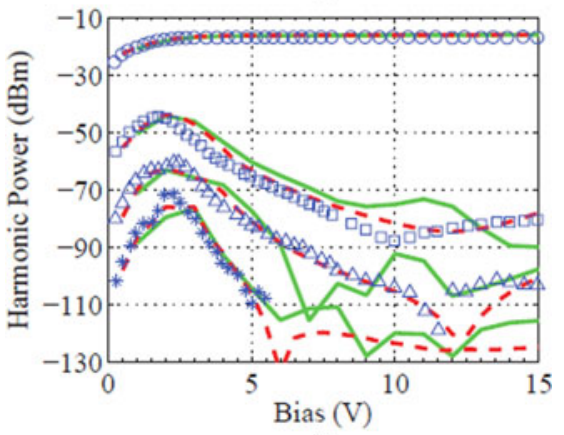

(b)

Fig. 11. Measured and calculated harmonic powers of the photodetector output current as a function of the reverse biases with (green solid curve) and without (red dashed curve) the Franz-Keldysh effect. Impact ionization and a $50 \Omega$ load resistor are kept in the simulations. (a) $1 \mathrm{D}$ model. (b) $2 \mathrm{D}$ model. 


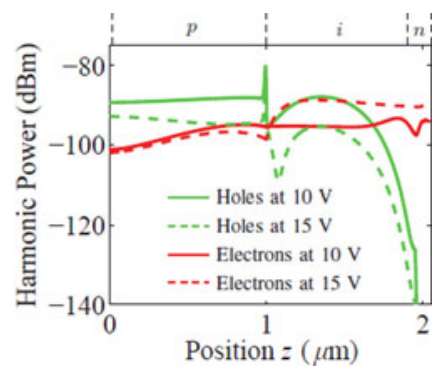

Fig. 12. Calculated hole and electron current contribution to the second harmonic power.

monic powers as the reverse bias increases. We conclude that the Franz-Keldysh effect does not play a visible role in this device. We note that the Franz-Keldysh effect is a quantum coherent effect that requires maintaining phase coherence of the electron wave functions, and we also note that the light wavelength of $1319 \mathrm{~nm}$ corresponds to an energy of $0.94 \mathrm{eV}$, which differs significantly from the band edge of $0.74 \mathrm{eV}$. We speculate that electron-phonon, electron-carrier, and/or electron-impurity collisions are disrupting the quantum coherence that is needed for the Franz-Keldysh effect to appear in the experimental devices. We note that the Franz-Keldysh effect has been observed to be important in some modern-day devices [11], [13].

\section{SOURCES OF NONLINEARITY IN THE PHOTODETECTOR}

Space charge was shown to be the dominant source of nonlinearity in a p-i-n photodetector when the reverse bias is low [2], [3], but its importance should decrease when the reverse bias increases. Since the harmonic power saturates and then typically begins to increase again beyond $10 \mathrm{~V}$, there must be another source of nonlinearity. We have shown in Fig. 10 that impact ionization is the source of that nonlinearity in our simulations. In order to determine the relative importance of the electron and hole contributions to the nonlinearity, we plot the hole and electron current contributions to the second harmonic power as a function of position $z$ in Fig. 12. We obtain the output current as a function of $z$ in the 1D model and 2D model. We then take the Fourier transform of the output current to obtain the harmonic power as a function of position $z$. We see that the electron current contribution to the second harmonic power decreases slowly in the p-region, and increases in the i-region when the reverse bias increases from 10 to $15 \mathrm{~V}$. However, the hole current contribution to the second harmonic power decreases when the reverse bias increases from 10 to $15 \mathrm{~V}$. So, impact ionization leads primarily to an increase in the electron current. At $10 \mathrm{~V}$, the holes dominate the nonlinear contribution. At $15 \mathrm{~V}$, in the p-region, the hole current contribution to the second harmonic power is larger than the electron current contribution; however, the electron current contribution is larger in the intrinsic region. In total, the hole and electron current contributions to the second harmonic power are almost the same. The hole velocity saturates more slowly than the electron velocity. So, a structure that limits the hole current will decrease the harmonic power. That may be done, for example, by inserting a p-doped absorber and an $\mathrm{n}$-doped absorber on each side of the intrinsic region to balance the carriers, as discussed by Tulchinsky et al. [29].

\section{CONCLUSION}

We studied 1D and 2D models of a single heterojunction $\mathrm{p}$ - $\mathrm{i}$ $\mathrm{n}$ photodetector that are based on the drift-diffusion equations. The harmonic power in these devices decreases from $2 \mathrm{~V}$ to about $10 \mathrm{~V}$, then saturates at around $10 \mathrm{~V}$, and then in most cases increases again as the reverse bias increases. We have examined the impact of an external load, thermionic emission at the $\mathrm{p}$ - $\mathrm{i}$ $\mathrm{n}$ interfaces, incomplete ionization, impact ionization, and the Franz-Keldysh effect at room temperature. We have found that the dominant physical cause of the observed saturation and the increase at large reverse bias is impact ionization in the simple $\mathrm{p}-\mathrm{i}-\mathrm{n}$ device that we are studying.

Hole and electron contributions to the second harmonic power were calculated. The hole current contribution to the second harmonic power decreases when the reverse bias increases from 10 to $15 \mathrm{~V}$, while the electron current contribution to the second harmonic power remains nearly constant in the p-region and increases slightly in the intrinsic region. Decreasing the hole injection will lead to a decrease in the harmonic power, which is consistent with the results of Tuchinsky et al. [29].

\section{ACKNOWLEDGMENT}

The authors would like to thank J. Diehl and M. Hutchinson for useful discussions. Work at UMBC was partially supported by the Naval Research Laboratory. The 2D simulations were carried out at UMBC's high performance computing facility.

\section{REFERENCES}

[1] M. Currie and I. Vurgaftman, "Microwave phase retardation in saturated InGaAs photodetectors," IEEE Photon. Technol. Lett., vol. 18, no. 13, pp. 1433-1435, Jul. 2006.

[2] K. J. Williams, R. D. Esman, and M. Dagenais, "Nonlearities in p-i-n microwave photodetectors," J. Lightw. Technol., vol. 14, pp. 84-96, 1996.

[3] K. J. Williams, "Microwave nonlinearities in photodiodes" $\mathrm{PhD}$ Dissertation, University of Maryland, College Park, MD, USA, 1994.

[4] G. M. Dunn, A. B. Walker, A. J. Vickers, and V. R. Wicks, "Transient response of photodetectors," J. Appl. Phys., vol. 79, pp. 7329-7338, 1996

[5] A. B. Walker and S. P. Wilson, "One- and two-dimensional models of the transient response of metal semiconductor metal photodetectors including diffraction," Semicond. Sci. Technol., vol. 12, pp. 1265-1272, 1997.

[6] H. Jiang and P. K. L. Yu, "Equivalent circuit analysis of harmonic distortions in photodiode," IEEE Photon. Technol. Lett., vol. 10, no. 11, pp. 1608-1610, Nov. 1998.

[7] K. Horio and H. Yanai, "Numerical modeling of heterojunctions including the thermionic emission mechanism at the heterojunction interface," IEEE Trans. Electron Devices, vol. 37, no. 4, pp. 1093-1098, Apr. 1990.

[8] B. K. Crone, P. S. Davids, I. H. Campbell, and D. L. Smith, "Device model investigation of bilayer organic light emitting diodes," J. Appl. Phys., vol. 87, pp. 1974-1982, 2000.

[9] K. Yang, J. C. Cowles, J. R. East, and G. I. Haddad, "Theoretical and experimental DC characterization of InGaAs-based abrupt emitter HBT's,' IEEE Trans. Electron Devices, vol. 42, no. 6, pp. 1047-1058, Jun. 1995.

[10] H. Wang and G.-I. Ng, "Avalanche multiplication in InP/InGaAs double heterojunction bipolar transistors with composite," IEEE Trans. Electron Devices, vol. 47, no. 6, pp. 1125-1133, Jun. 2000.

[11] Y. Fu, H. Pan, Z. Li, A. Beling, and J. C. Campbell, "Characterizing and Modeling Nonlinear Intermodulation Distortions in Modified Unitraveling Carrier Photodiodes," IEEE J. Quantum Electron., vol. 47, no. 10, pp. 1312-1319, Oct. 2011. 
[12] Y. Hu, C. R. Menyuk, V. J. Urick, and K. J. Williams, "Sources of nonlinearity in a PIN photodetector at high applied reverse bias," in Proc. IEEE Int. Topical Meet. Microw. Photon., 2013, pp. 282-285.

[13] A. S. Hastings, D. A. Tulchinsky, K. J. Williams, H. Pan, A. Beling, and J. C. Campbell, "Minimizing photodiode nonlinearities by compensating voltage-dependent responsivity effects," J. Lightw. Technol., vol. 28 , pp. 3329-3333, 2010.

[14] S. Selberherr, Analysis and Simulation of Semiconductor Devices. New York, NY, USA: Springer, 1984.

[15] M. Dentan and B. de Cremoux, "Numerical simulation of the nonlinear response of a p-i-n photodiode under high illumination," J. Lightw. Technol., vol. 8, pp. 1137-1144, 1990.

[16] K. Williams, R. Esman, R. Wilson, and J. Kulick, "Differences in p-side and n-side illuminated p-i-n photodiode nonlinearities," IEEE Photon. Technol. Lett., vol. 10, no. 1, pp. 132-134, Jan. 1998.

[17] S. P. Wilson, "Modelling of p-i-n photodiodes under high illumination conditions," Int. J. Numer. Modell. Electron. Netw. Devices Fields, vol. 10 , pp. 139-151, 1997.

[18] S. J. Woods, S. P. Wilson, and A. B. Walker, "Non-unique solutions in drift diffusion modelling of phototransistors," Int. J. Numer. Modell. Electron. Netw. Devices Fields, vol. 13, pp. 37-57, 2000.

[19] S. A. Malyshev, A. L. Chizh, and Y. G. Vasileuski, "2D Simulation of planar InP/InGaAs avalanche photodiode with no guard rings," in Proc. Int. Conf. Numer. Simul. Optoelectron. Devices, 2008, pp. 81-82.

[20] A. Habibpoor and H. Mashayekhi, "Numerical modeling of the effect of optical pulse position on the impulse response of a Metal-SemiconductorMetal (MSM) photodetector (low field condition)," Eur. Phys. J. Appl. Phys., vol. 55, p. 10502, 2011.

[21] A. E.-N. A. Mohamed, N. A. Ayad, A. Nabih, Z. Rashed, and H. M. ElHageen, "Speed response and performance degradation of high temperature gamma irradiated silicon PIN photodiodes," Int. J. Com. Sci. Telecom., vol. 2, pp. 15-22, 2011.

[22] K. W. Boer, Survey of Semiconductor Physics. New York, NY, USA: Van Nostrand Reinhold, 1990.

[23] C. Hilsum, "Simple empirical relationship between mobility and carrier concentration," Electron. Lett., vol. 10, pp. 259-260, 1974.

[24] D. Hahn, O. Jaschinski, H. H. Wehmann, and A. Schlachetzki, "Electron-Concentration dependence of absorption and refraction in $\mathrm{n}$ $\mathrm{In}_{0.53} \mathrm{Ga}_{0.47}$ As near the band-edge," J. Elec. Mater., vol. 24, pp. 1357$1361,1995$.

[25] T. P. Pearsall, "Impact ionization rates for electrons and holes in $\mathrm{Ga}_{0.47} \mathrm{In}_{0.53}$ As," Appl. Phys. Lett., vol. 36, pp. 218-220, 1979.

[26] J. Marsland, R. Woods, and C. Brownhill, "Lucky drift estimation of excess noise factor for conventional avalanche photodiodes including the dead space effect," IEEE Trans. Electron. Devices, vol. 39, no. 5, pp. 11291135, May 1992.

[27] J. Callaway, "Optical absorption in an electric field," Phys. Rev., vol. 130, pp. 549-553, 1963.

[28] B. O. Seraphin and N. Bottka, "Franz-Keldysh effect of the refractive index in semiconductors," Phys. Rev., vol. 139, pp. A560-A565, 1965.

[29] D. A. Tulchinsky, X. Li, N. Li, S. Demiguel, J. C. Campbell, and K. J. Williams, "High-saturation current wide-bandwidth photodetectors," IEEE J. Sel. Topics Quantum Electron., vol. 10, no. 4, pp. 702-708, Jul./Aug. 2004.

Yue Hu (S'12) was born on in China in 1985. He received the B.S. degree in electrical science and technology from University of Electrical Science and Technology of China, Chengdu, China, in 2006. He is currently working toward the Ph.D. degree with the Department of Computer Science and Electrical Engineering, University of Maryland Baltimore County, Baltimore, MD, USA

His current research interests include nonlinearity sources in high-power photodetectors.

Brian Marks (M'01) received the Bachelor's degree in mathematics and physics from North Carolina State University, Raleigh, NC, USA, in 1995, and the Ph.D. degree in engineering sciences and applied mathematics from Northwestern University, Chicago, IL, USA, in 2000 . He subsequently served in positions in the Department of Computer Science and Electrical Engineering, University of Maryland Baltimore County, Baltimore, MD, USA, as a Research Assistant Professor and at Indiana University, Bloomington, IN, USA, as a mathematics and statistics Lecturer. Since 2013, he has been a Member of the professional staff at the Johns Hopkins University Applied Physics Laboratory, Laurel, MD, USA.
Curtis R. Menyuk (F'98) was born in March 26, 1954. He received the B.S. and M.S. degrees from the Massachussets Institute of Technology, Cambridge, MA, USA, in 1976, and the Ph.D. degree from the University of California Los Angeles, Los Angeles, CA, USA, in 1981. He has worked as a Research Associate at the University of Maryland, College Park, MD, USA, and at Science Applications International Corporation in McLean, VA, USA. In 1986, he became an Associate Professor in the Department of Electrical Engineering, University of Maryland Baltimore County (UMBC), Baltimore, MD, USA, and he was the Founding Member of this department. In 1993, he was promoted to Professor. He was on partial leave from UMBC from Fall, 1996 until Fall, 2002. From 1996 to 2001, he worked part-time for the Department of Defense, co-directing the Optical Networking program at the DoD Laboratory for Telecommunications Sciences in Adelphi, MD, from 1999 to 2001. From 2001-2002, he was the Chief Scientist at PhotonEx Corporation, Maynard, MA. From 2008 to 2009, he was a JILA Visiting Fellow at the University of Colorado, Boulder, CO, USA. For the last 25 years, his primary researchinterest has been theoretical and computational studies of lasers, nonlinear optics, and fiber optic communications. He has authored or co-authored more than 240 archival journal publications as well as numerous other publications and presentations, and he is a co-inventor of five patents. He has also edited three books. The equations and algorithms that he and his research group at UMBC have developed to model optical fiber systems are used extensively in the telecommunications and photonics industry. $\mathrm{He}$ is a Member of the Society for Industrial and Applied Mathematics and of SPIE. He is a Fellow of the American Physical Society and the Optical Society of America. He is a former UMBC Presidential Research Professor. Dr. Menyuk received the 2013 IEEE Photonics Society William Striefer Award.

Vincent J. Urick (M'05-SM'12) received the B.S. degree (magna cum laude) in physics with minors in electronics and mathematics from Bloomsburg University, Bloomsburg, PA, USA, in 2001, and the M.S. and Ph.D. degrees from George Mason University, Fairfax, VA, USA, in 2005 and 2007, respectively. In 2001, he joined the US Naval Research Laboratory (NRL), Washington, DC, USA, as a Research Physicist in the Photonics Technology Branch. He is currently the Head of the Applied RF Photonics Section at NRL directing and conducting research in microwave photonics, a field where he has more than 90 publications. His current research interests include novel applications of photonics technology. Dr. Urick was awarded the Department of the Navy Top Scientists and Engineers of the Year Award in 2007 for his work on long-haul analog delay lines. He serves or has served on the Technical Program Committees for the IEEE Avionics Fiber Optics and Photonics Conference, the IEEE International Meeting on Microwave Photonics and the IEEE Photonics Conference, and the 2013 Organizing Committee for the IEEE International Meeting on Microwave Photonics. He is a Member of the IEEE Photonics Society Technical Advisory Council and chairs the Microwave Photonics Committee for the IEEE Photonics Conference.

Keith J. Williams (S'86-M'89) received the B.S. degree (cum laude) in electrical engineering from the University of Nebraska, Lincoln, NE, USA, in 1987, and the M.S. and Ph.D. degrees in electrical engineering from the University of Maryland, College Park, MD, USA, in 1989 and 1994, respectively. His doctoral dissertation research concerned the area of microwave $p$-i- $n$ photodetector nonlinearity.

In 1987, he joined the Optical Sciences Division, Naval Research Laboratory, Washington, DC, USA, where his research interests include characterization and performance of microwave optical devices, microwave BER-optic links and systems, high-speed optoelectronics, new concepts for solving microwave related problems with fiber-optic solutions, and high current photodiodes.

Beta $\mathrm{Pi}$

Dilliams is a Member of the Optical Society of America and Tau 